\title{
Geschlechtsspezifische Unterschiede beim Lungenkarzinom
}

\author{
Gender-Specific Difference in Lung Cancer
}

Autoren

Institut
M. Serke, F. Stanzel, M. Westhoff

Pneumologie, Lungenklinik Hemer $\begin{array}{ll}\text { eingereicht } & \text { 12. 2. } 2013\end{array}$ akzeptiert nach Revision 22. 3.2013

Bibliografie DOI http://dx.doi.org/ 10.1055/s-0033-1343149 Pneumologie 2013; 67: 270-279 (c) Georg Thieme Verlag KG Stuttgart · New York ISSN 0934-8387

Korrespondenzadresse Dr. med. Monika Serke Pneumologie, Lungenklinik Hemer

Theo-Funccius-Str. 1 58675 Hemer

monika.serke@lkhemer.de

Serienherausgeber C. Grohé, Berlin

\section{Zusammenfassung \\ $\nabla$}

Epidemiologisch zeigt sich in Deutschland, in Europa und in den USA eine zunehmende Lungenkrebshäufigkeit bei Frauen! Besonders der Vergleich der altersspezifischen Erkrankungsraten nach Geschlecht zeigt bei jüngeren Frauen eine höhere Lungenkrebserkrankungsrate als bei Männern. Histologisch sind bei Frauen mehr Adenokarzinome als bei Männern zu beobachten. Ursächlich sind einerseits sicherlich die geänderten Rauchgewohnheiten der Frauen, zusätzlich spielen aber noch andere Faktoren bei der Lungenkrebs-Karzinogenese der Frauen eine Rolle. Wesentlich häufiger manifestiert sich der Nichtraucher-Lungenkrebs bei Frauen als bei Männern. Ursächlich könnten unterschiedliche Lungenkrebs-Empfänglichkeiten oder auch unterschiedliche Karzinogen-Expositionen, z.B. gegenüber Radon, durch Passivrauchen oder Herddämpfe der Geschlechter sein. Auf molekularer Ebene finden sich bei Frauen häufiger genetische „TreiberMutationen“ als bei Männern und demzufolge können neue „zielgerichtete Therapien“, z.B. gegen EGFR oder Alk, häufiger bei Frauen zum Einsatz kommen. Die Prognose beim klein- und nichtkleinzelligen Lungenkarzinom ist bei Frauen insgesamt etwas besser als bei Männern.

\section{Einleitung}

\section{$\checkmark$}

Lungenkrebs, in den ersten Jahrzehnten des letzten Jahrhunderts noch selten bei Frauen, hat in den letzten 30 Jahren bei Frauen um das Vierfache an Häufigkeit zugenommen und entwickelte sich somit beim weiblichen Geschlecht zu einer schleichenden und tödlichen Epidemie!

Diese epidemiologische Entwicklung und die Entdeckung der molekular gesteuerten Therapien mit der damit verbundenen Identifikation der Zielgruppe der weiblichen Nichtraucher bewirkte beim Lungenkarzinom ein zunehmendes Interes-

\section{Abstract \\ $\nabla$}

More and more differences in lung cancer are being detected between men and women. Lung cancer, at the beginning of the last century a rare disease in women, has a growing incidence in women, in particular in young females. Lung cancer is a leading cause of cancer death in women in developed countries with different histological types and adenocarcinomas are more frequent in women than in men. Cigarette smoking is the most prevalent cause of lung cancer in women, in addition susceptibility to carcinogens may differ between the sexes. As more non-smoking women than men develop lung cancer, it is likely that they are exposed to excessive environmental carcinogens such as second-hand-smoking, inhouse-radon or cooking fumes. Furthermore, genetic and hormonal influences play a role in lung cancer etiology for women. Taken together, women have a better overall survival than men with lung cancer. Differences in molecular susceptibility patterns are observed between men and women, and show that molecular targets such as EGFR or ALK more frequent in women.

se an der Unterscheidung der Patienten nach Geschlecht und Raucherstatus. Auch zeigten Therapiestudien häufig eine bessere Prognose bei Frauen.

\section{Epidemiologie Europa und USA: zunehmend bei Frauen}

$\nabla$

Die Lungenkrebsmortalität nimmt in der EU bei Männern ab. Europäischer Spitzenreiter der Häufigkeit, an Lungenkrebs zu erkranken, ist Polen, am niedrigsten ist in Europa die Lungenkrebshäu- 


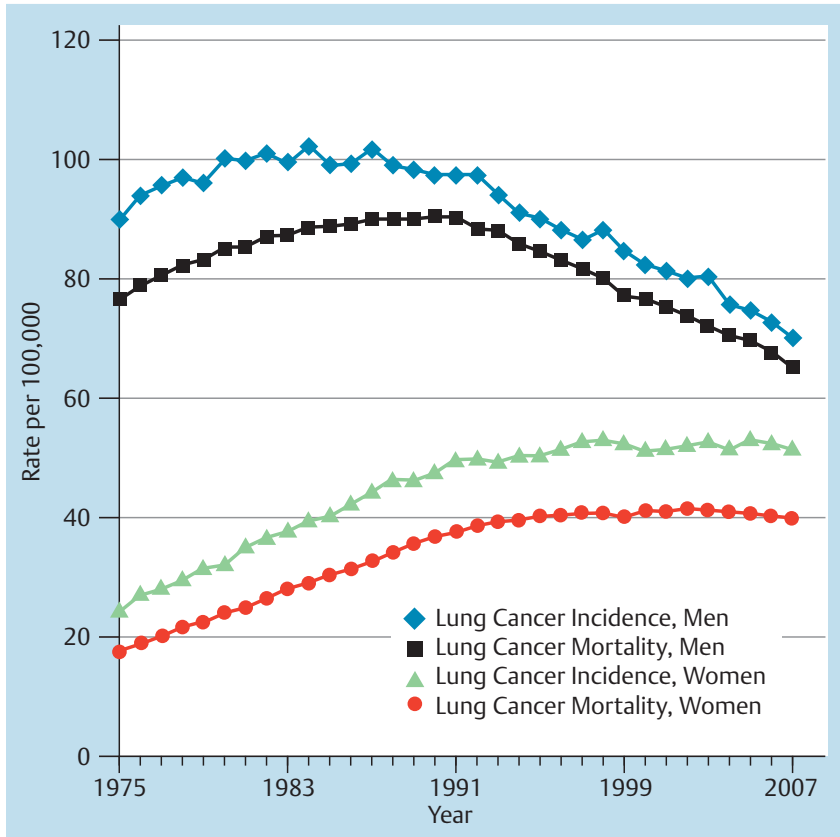

Abb.1 Altersadjustierte Lungenkrebs-Inzidenz und Mortalität bei Männern und Frauen 1975-2007 [10].

figkeit in England und Deutschland, sie liegt in Spanien und Frankreich höher als in Italien. Im Gegensatz zu den Männern nimmt die Lungenkrebshäufigkeit von Frauen seit 2007 in der EU zu. Es wird eine zunehmende Lungenkrebshäufigkeit bei Frauen in der EU erwartet mit einer Steigerung der Inzidenz von 14-15 auf 100000 im Jahr 2015. Bereits 2007-2008 war Lungenkrebs die häufigste Krebserkrankung bei Frauen in England und Polen und häufiger als Brustkrebs [1].

USA: Seit Jahrzehnten ist das Lungenkarzinom in den USA die häufigste Krebs-Todesursache bei Männern. Neu manifestierte sich jetzt eine zunehmende Lungenkrebshäufigkeit bei Frauen. Seit 1988 wurde das Lungenkarzinom in den USA auch bei Frauen die häufigste krebsbedingte Todesursache [2] ( $\bullet$ Abb.1). In den USA sterben jährlich mehr Frauen an Lungenkrebs als an Mamma-, Ovarial- und Zervixkarzinom zusammen [3,4].

Eine aktuelle Analyse [5] zeigte, dass das relative und absolute Risiko eines Zigaretten-bedingten Todes bei weiblichen Rauchern in den letzten Jahren deutlich zugenommen hat. Das relative Risiko, an Lungenkrebs, COPD, koronarer Herzkrankheit oder Schlaganfall zu sterben ist jetzt für männliche und weibliche Raucher gleich groß. Diese Beobachtung bestätigt die Hypothese, dass „Frauen, die wie Männer rauchen, auch wie Männer sterben“.

\section{Ethnische Faktoren USA}

Die Herkunft bedingt unter Rauchern bei Männern und Frauen, auch unter Berücksichtigung der Intensität des Nikotinkonsums, eine unterschiedliche Lungenkrebshäufigkeit. Unter Rauchern ist bei Afroamerikaner der Lungenkrebs häufiger als bei weißen
Tab. 1 5-Jahres-Überleben nach Geschlecht und Histologie [10]

\begin{tabular}{|lll|}
\hline Histologie & $\begin{array}{l}\text { 5-J-ÜL Männer \% } \\
\text { alle Stadien }\end{array}$ & $\begin{array}{l}\text { 5-J-ÜL Frauen \% } \\
\text { alle Stadien }\end{array}$ \\
\hline Adeno & 17,1 & 23,7 \\
\hline Plattenepithel-CA & 16,3 & 18 \\
\hline großzellig & 11,4 & 13 \\
\hline kleinzellig & 5,1 & 7,1 \\
\hline andere & 11,7 & 18,4 \\
\hline
\end{tabular}

Amerikanern, Japanisch-stämmigen Amerikanern oder Latinos [6]. Auch bei Frauen ist die Lungenkrebsinzidenz am höchsten bei Afroamerikanerinnen mit 161/100000, gefolgt von weißen Amerikanerinnen (133/100000) und deutlich niedriger bei Latinos (47/100000) oder japanisch-stämmigen Amerikanerinnen (50/100000). Afroamerikanische Frauen erkranken häufiger an Lungenkrebs als weiße Frauen, obwohl in den USA mehr weiße (20\%) als afroamerikanische Frauen (17\%) rauchen.

Ursächlich könnten ethnisch bedingte Variationen im Nikotinmetabolismus oder auch unterschiedliche Rauchgewohnheiten und Karzinogen-Expositionen sein.

\section{Epidemiologie Deutschland}

In Deutschland ist Lungenkrebs die dritthäufigste Krebserkrankung bei Männern und Frauen [7]. Das Lungenkarzinom tritt bei Männern aber immer noch häufiger auf als bei Frauen: 2008 erkrankten in Deutschland rund 34000 Männer und 15500 Frauen an Lungenkrebs, ca. 29500 Männer und 13000 Frauen verstarben daran [7]. Die insgesamt schlechte Prognose unterscheidet sich geringfügig zwischen Männern und Frauen zuungunsten der Männer. So liegt die 5-Jahres-Überlebensrate bei Männern bei 15\% und beträgt bei Frauen 18\% ( Tab. 1). In der Krebs-Mortalität steht das Lungenkarzinom in Deutschland bei Männern auf dem ersten und bei Frauen auf dem 3. Platz. Die Stadienverteilung unterscheidet sich nicht zwischen Männern und Frauen. Das mittlere Erkrankungsalter liegt bei etwa 69 Jahren für Männer und bei 68 Jahren für Frauen.

Über die Jahre zeigt sich in Deutschland bei Männern und Frauen ein gegenläufiger Trend der Erkrankungs- und Sterberaten: Bei den Frauen wurde das Lungenkarzinom seit Ende der 1990erJahre um etwa 30\% häufiger, bei den Männern hingegen gingen die Erkrankungsraten kontinuierlich zurück. Der Vergleich der altersspezifischen Erkrankungsraten nach Geschlecht zeigt in den jüngeren Lebensaltern, d. h. unter 44 Jahren, eine altersspezifische höhere Lungenkrebserkrankungsrate bei Frauen als bei Männern ( Tab.2).

\section{Rauchgewohnheiten von Männern und Frauen weltweit und in Deutschland}

Wichtigster Risikofaktor für das Lungenkarzinom ist das Zigarettenrauchen mit klarer Dosis-Wirkungsbeziehung zwischen dem Rauchen und dem steigenden Lungenkrebsrisiko in Abhängigkeit von Packungsjahren und Alter beim Rauchbeginn. Rauchgewohnheiten von Männern und Frauen weltweit sind abhängig

Tab.2 Altersspezifische Erkrankungsraten nach Geschlecht, ICD-10 C33 - 34, Deutschland, 2007 - 2008 je 100000 [7].

\begin{tabular}{llllllllllllllll}
$\begin{array}{l}\text { Alter| } \\
\text { Jahre }\end{array}$ & $\mathbf{1 5 - 1 9}$ & $\mathbf{2 0 - 2 4}$ & $\mathbf{2 5 - 2 9}$ & $\mathbf{3 0 - 3 4}$ & $\mathbf{3 5 - 3 9}$ & $\mathbf{4 0 - 4 4}$ & $\mathbf{4 5 - 4 9}$ & $\mathbf{5 0 - 5 4}$ & $\mathbf{5 5 - 5 9}$ & $\mathbf{6 0 - 6 4}$ & $\mathbf{6 5 - 6 9}$ & $\mathbf{7 0 - 7 4}$ & $\mathbf{7 5 - 7 9}$ & $\mathbf{8 0 - 8 4}$ & $\mathbf{7 8 5}$ \\
\hline Männer & 0,2 & 0,3 & 1,3 & 0,8 & 3,2 & 7,9 & 28,2 & 66,1 & 119,1 & 189,5 & 259,6 & 329,2 & 422,0 & 480,6 & 332,9 \\
\hline Frauen & 0,3 & 0,3 & 0,3 & 1,5 & 3,4 & 8,3 & 20,5 & 41,1 & 61,5 & 85,1 & 87,8 & 97,1 & 119,6 & 116,3 & 92,3 \\
\end{tabular}




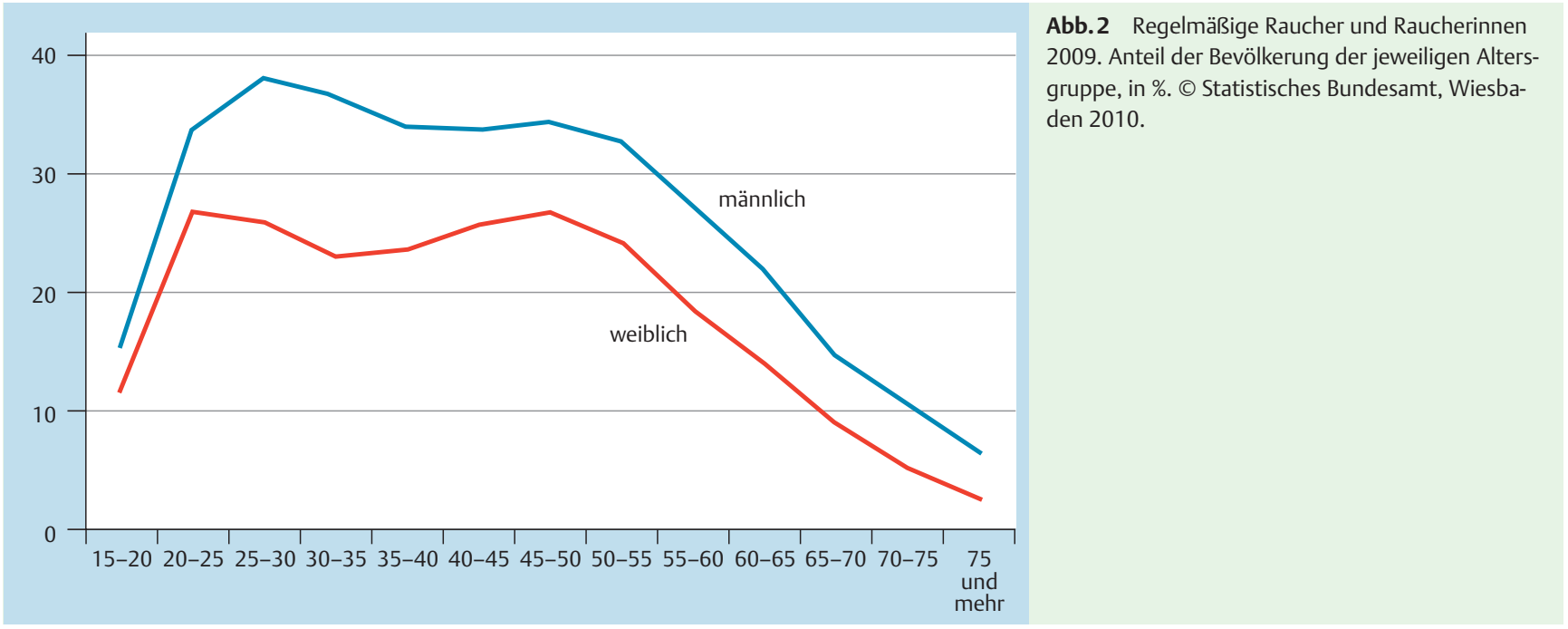

von den regionalen, sozialen und kulturellen Bedingungen, und sie sind sehr unterschiedlich. Weltweit rauchen Frauen mit einer sehr unterschiedlichen Inzidenz von $0,6-35,6$ per 100000 . In den USA und in Europa nahm der Nikotinkonsum der Frauen [8] seit den 40 -50er Jahren deutlich zu, seit den 80er Jahren zeichnet sich ein langsamer Rückgang des Rauchens ab. Allerdings betrug die Raucherquote bei Frauen in den USA 2004 immer noch alarmierende $22 \%$.

Das Rauchen ist vermutlich weltweit verantwortlich für $71 \%$ der Lungenkarzinome, $42 \%$ der chronischen Atemwegserkrankungen und $10 \%$ der kardiovaskulären Erkrankungen. Weltweit rauchen viel mehr Männer als Frauen, besonders in den ärmeren Regionen der Welt. Dennoch ist Zigarettenrauch verantwortlich für 6\% der weiblichen Todesfälle [9], und es muss berücksichtigt werden, dass weltweit, besonders in ärmeren Ländern, das Zigarettenrauchen der Frauen zunimmt [10].

Unter den rauchenden Frauen in England nimmt man an, dass 2/3 aller Todesfälle im Alter zwischen 50-80 Jahren Zigarettenassoziiert sind. Raucherinnen verlieren durchschnittlich 10 Lebensjahre durch das Rauchen. Diese Risiken können sich verringern, wenn möglichst früh, am besten vor dem 40. Lebensjahr das Rauchen eingestellt werden kann [11].

Im Jahr 2009 wurden in Deutschland knapp 87 Milliarden Zigaretten, rund 3,8 Milliarden Zigarren oder Zigarillos, über 24000 Tonnen Feinschnitt und gut 800 Tonnen Pfeifentabak versteuert [12]. Dies entspricht einem durchschnittlichen Konsum von drei Zigaretten täglich pro Kopf der Bevölkerung-vom Säugling bis zum Greis. Nach den Ergebnissen des Mikrozensus war im Jahr 2009 ein Viertel der Personen ab 15 Jahren bekennender Raucher. 79\% der Frauen und 69\% der Männer bezeichneten sich im Jahr 2009 als Nichtraucher.

Betrachtet man das Alter der Raucher, zeigt sich der Spitzenwert der Raucher in den jüngeren Altersgruppen: bei den Männern bei den 25- bis 30-Jährigen, bei den Frauen früher mit den meisten Raucherinnen im Alter zwischen 20-25 Jahre. Bei den Jugendlichen und jungen Erwachsenen von 15 bis unter 20 Jahren ist der Anteil der Raucher seit 1999 rückläufig und sank bei den Männern von 22 auf $16 \%$ und bei den Frauen von 16 auf $12 \%$ [12] $($ Abb.2)

Der Zeitpunkt des Rauchbeginns liegt bei jungen Frauen früher als bei Männern: 15- bis unter 20-jährige Männer geben ihren Rauchbeginn mit 15,6 Jahren an, die gleichaltrigen Frauen mit 15,2 Jahren. Auch in der Altersgruppe der 20- bis unter 25-Jähri- gen begannen die Frauen etwas früher zu rauchen mit durchschnittlich 15,7 Jahren als die gleichaltrigen Männer mit 16,0 Jahren (12 Laue 2010). Männer rauchen intensiver als Frauen: Von den Rauchern bezeichnete sich jeder Sechste (17\%) als starker Raucher, von den Raucherinnen nur jede Zehnte (10\%). Der Anteil der Frauen, die weniger als 5 Zigaretten pro Tag rauchten, ist mit $9 \%$ fast doppelt so hoch wie bei Männern mit 5\%.

\section{Höhere Empfindlichkeit der Frauen auf das Rauchen? $\nabla$}

Man vermutet schon länger, dass bei gleichem Zigarettenkonsum Frauen ein höheres Lungenkrebsrisiko als Männer haben. Bei Frauen mit 40 Packungsjahren war die Wahrscheinlichkeit, ein Lungenkarzinom [13] zu entwickeln 28-mal so hoch wie bei nicht rauchenden Frauen und 3-mal so hoch wie bei rauchenden Männern.

So fanden sich in der großen „Nurses Health Study/Health Professionals' Follow-up Study“ bei weiblichen Rauchern höhere Lungenkrebsinzidenzen als bei männlichen Rauchern [14]. Die ELCAP-Lungenkrebsscreening-Studie, durchgeführt bei Probanden über 40 Jahre mit mindestens 10 Packungsjahren, zeigte beim ersten Screening-CT nach Abgleich von Alter und Packungsjahren ein bei Frauen um 2,7-fach erhöhtes Risiko und eine bei Frauen höhere Lungenkrebsprävalenz [15] als bei vergleichbar rauchenden Männern. Die Detektionsrate bei Frauen war mit $2,08 \%$ doppelt so hoch wie mit 1,02\% bei Männern. Hingegen war das Überleben bei Frauen besser (HR: 0,48), vielleicht, weil bei Frauen mehr frühe, gut kurativ operable, gut differenzierte Adenokarzinome entdeckt wurden. Henschke stellte die Hypothese auf, dass Frauen empfindlicher für das Lungenkarzinom sind als Männer, aber beim Lungenkarzinom ein besseres Überleben als Männer haben.

In Korea [16] erkrankten rauchende Frauen nach weniger Packungsjahren als die Männer und hatten stärkere Einschränkungen der Lungenfunktion. Ein sog. „Empfindlichkeits-Index“ war für Frauen signifikant höher als für Männer. In Korea rauchen aber nur 2\% der Frauen, und die Zahl der in die Studie eingeschlossenen Frauen war klein. Möglicherweise bedingt das kleinere Lungenvolumen der Frauen im Vergleich zu den Männern, bezogen auf den gleichen Zigarettenkonsum, die höhere Empfindlichkeit der Frauen. 
In den USA beobachtete man bei Frauen eine gering höhere Lungenkrebsinzidenz, sowohl bei Nie-Rauchern als auch bei Rauchern: Nie-rauchende Frauen hatten eine Lungenkrebsinzidenz von 25 auf 100000, nie-rauchende Männer 20 auf 100000, bei Rauchern und Raucherinnen mit mehr als 2 Packungen/Tag war die Inzidenz deutlich höher: 1260 auf 100000 bei Männern und 1310 bei Frauen [17].

Zusammenfassend scheint die Empfindlichkeit der Frauen gegenüber den karzinogenen Effekten des Zigarettenrauchs höher als die der Männer zu sein, bedingt durch genetische, hormonelle oder metabolische Faktoren, allerdings gibt es in dieser Frage in der Literatur auch die Meinung, dass kein Empfindlichkeitsunterschied zwischen den Geschlechtern bestehe. Bain [18] beobachtete die Lungenkrebshäufigkeit an über 80000 Männern und Frauen und fand keinen Unterschied im Lungenkrebsrisiko zwischen Männern und Frauen.

\section{Unterschiedliche Reparatur-Gen-Ausstattung bei Männern und Frauen? \\ $\nabla$}

Zigarettenrauch enthält ein Gemisch von über 100 Mutagenen und Karzinogenen, die alle eine Wirkung auf DNA und die Zellteilung haben können. Auf molekularer Ebene werden verschiedene geschlechtsbezogene Mechanismen diskutiert [19]. Zum Beispiel wird eine verminderte DNA-Reparaturkapazität (DRC) bei Lungenkrebspatienten als möglicher Auslöser für Lungenkrebs diskutiert. Frauen mit Lungenkrebs hatten eine erniedrigte DRC im Vergleich zu Männern und gesunden Frauen. Diese verringerte DNA-Reparaturkapazität fand sich unabhängig von Nikotinkonsum bei Frauen mit Lungenkrebs und scheint eine Zigarettenrauch-unabhängige Lungenkrebsursache zu sein $[20,21]$. Bei Frauen wurden höhere Spiegel von Zigarettenrauch-induzierten DNA-Schäden im Lungengewebe gefunden als bei Männern [22]. Man fand bei rauchenden Frauen eine erhöhte Expression von CYP1A-Genen in der Lunge, verglichen mit männlichen Rauchern. Diese könnten erhöhte Spiegel von DNA-Addukten bewirken und damit die Möglichkeit verringern, Zigaretten-Karzinogene zu entgiften [23]. Diese erhöhte CYP1-Expression könnte hormonabhängig, speziell östrogenabhängig, sein.

Ein häufiger Polymorphismus im Bereich der DetoxifizierungsEnzyme betrifft die Gluthation S-Transferase M1 (GSTM1), die bei 40-60\% der Bevölkerung, bedingt durch eine Gen-Deletion, vorliegt. In Kombination mit einer hohen CYP1A-Genexpression steigt das Lungenkarzinomrisiko bei Frauen deutlich mehr als bei Männern (OR 6,54 vs. 2,3) [24].

Auch geschlechtsbezogene Unterschiede beim auf dem X-Chromosom lokalisierten Gastrin-releasing Peptid-Rezeptor (GRPR)Gen [25] könnten eine unterschiedliche Karzinogen-Empfindlichkeit bewirken.

\section{Passivrauchen}

$\nabla$

Das Passivrauchen ist einer der Risikofaktoren für das Lungenkarzinom und bei Frauen häufiger als bei Männern [26]. Weltweit sind im Jahr 2004 40\% der Kinder, 33\% der männlichen Nichtraucher und 35\% der weiblichen Nichtraucher gegenüber Passivrauch (second-hand smoke) exponiert. Man vermutet, dass diese Exposition 379000 Todesfälle durch ischämische Herzkrankheiten und 21400 durch Lungenkrebs ausgelöst hat, insgesamt geschätzt $1 \%$ der weltweiten Mortalität [27]. Geschätzte $47 \%$ der
Todesfälle durch Passivrauchen kommen bei Frauen, 28\% bei Kindern und 26\% bei Männern vor.

Nichtrauchende Ehefrauen von Rauchern haben ein um 24\% erhöhtes Lungenkrebsrisiko [28], verglichen mit nicht rauchenden Frauen ohne Passiv-Rauch-Exposition mit einer direkten Abhängigkeit zur Intensität des Passivrauchens. In einer Metaanalyse aus 19 Studien wird das Risiko Nie-Rauchender Ehefrauen durch das Passivrauchen um 20\% erhöht [29]. Neben dem Lungenkrebsrisiko erhöht das Passivrauchen auch das Risiko für Asthma, Emphysem und Atemwegsinfekte [30].

\section{Lungenkrebs bei Nichtrauchern (LCINS)}

$\nabla$

Bei Männern lassen sich in Deutschland neun von zehn, bei Frauen nur sechs von zehn Lungenkrebserkrankungen auf das aktive Rauchen zurückzuführen [7], d.h. es gibt mehr weibliche als männliche Nichtraucher unter den Lungenkrebspatienten. In den USA treten 10\% der Lungenkrebserkrankungen bei Nie-Rauchern auf (Lung cancer in never smokers: LCINS), als „Nie-Raucher-Lungenkrebs“. Hiervon sind Frauen überproportional häufiger betroffen als Männer. Unter amerikanischen Lungenkrebspatienten ist die Nichtraucher-Rate bei Frauen 2,5-fach so hoch wie bei Männern (15\% Frauen versus 6\% Männer) [31]. Offenbar ist dies ein Lungenkarzinom mit spezieller Pathogenese und anderen molekularen Veränderungen als bei Rauchern.

In Singapur [32] wurden Lungenkrebspatienten in Bezug auf Unterschiede zwischen Rauchern und Nie-Rauchern untersucht. Die Rate an Nie-Rauchern betrug 32\%, häufig waren Nie-Raucher $(68,5 \%)$ weiblich. Histologisch hatte Nichtraucher mehr Adenokarzinome $(69,9 \%)$ als Raucher $(39,9 \%)$ und deutlich weniger (5,9\%) Plattenepithelkarzinome als Raucher (35,7\%). Nie-Raucher waren jünger und wurden in fortgeschritteneren Stadien diagnostiziert. Das 5-Jahres-Überleben der Raucher war mit 7,7\% signifikant schlechter als das der Nie-Raucher, deren 5-Jahres-Überleben 10,8\% betrug. In dieser Studie war nicht das Geschlecht, sondern nur der Raucherstatus ein Prognosefaktor. Bei den Nichtrauchern bestanden zwischen Männern und Frauen große Ähnlichkeiten im klinischen Erscheinungsbild [32].

Das Adenokarzinom ist die häufigste Histologie beim LCINS [33], und beim LCINS finden sich häufiger aktivierende EGFR-Genmutationen. Das erklärt z.T. die guten Ansprech- und Überlebensraten bei Nie-Rauchern mit fortgeschrittenen NSCLC unter der EGFR-TKI-Therapie.

In einer japanischen Registerstudie [34] an 4954 NSCLC-Patienten im Stadium IIIB oder IV unter Chemotherapie fanden sich 149 Nie-Raucher und 3455 Raucher. Nie-Raucher waren signifikant häufiger weiblich, jünger, in besserem Allgemeinzustand und hatten häufig eine Adenokarzinom-Histologie.

Ein Vergleich an 650 monozentrisch beobachteten Patienten zeigte für Nichtraucher ein insgesamt besseres 5-Jahres-Überleben (23\%) gegenüber Rauchern mit einem 5-Jahres-Überleben von nur $16 \%$ [35].

\section{Zigaretten-unabhängige Risikofaktoren: Lungenkrebs bei nicht rauchenden Frauen \\ $\nabla$}

Die Lungenkrebshäufigkeit ist bei nicht rauchenden Frauen höher als bei nicht rauchenden Männern [33]: Altersadjustierte Inzidenzraten für das Lungenkarzinom bei Nie-Rauchern zwischen 40 - 79 Jahren sind für Frauen höher als für Männer: 14.4-20.8/ 
100000 Personen-Jahre bei Frauen und 4.8 - 13.7/100 000 Personen-Jahre bei Männern.

Zigaretten-unabhängige Risikofaktoren können Expositionen gegenüber Radon, Küchenabgasen [19], Asbest, Schwermetallen, Passivrauchen, Virusinfektionen (human papillomavirus infection) oder hormonelle Einflüsse sein. Man vermutet eine individuell gesteigerte genetische Empfänglichkeit, ohne dass für den Nie-Raucher-Lungenkrebs (LCINS) hierzu bisher wesentliche Informationen vorliegen.

Etwa 9-15 von 100 Lungenkrebsfällen werden in Deutschland auf berufliche kanzerogene Stoffe [7] wie Asbest und Quarzstäube, Dieselabgabe, polyzyklische aromatische Kohlenwasserstoffe und Nickelstäube zurückgeführt. Diese kommen vor allen an $\mathrm{Ar}-$ beitsplätzen von Männern vor. Hauswirtschaftlich tätige Frauen können durch die natürliche Radonbelastung von Gebäuden, besonders in den unteren Stockwerken einem erhöhten Lungenkrebsrisiko unterliegen [36]. Auch die Exposition gegenüber Koch- und Herdabgasen oder beim Kochen auftretende Öldämpfe [37] können Frauen mit inhalativen Karzinogenen belasten.

Chronische Lungenerkrankungen wie COPD, Silikose und idiopathische Lungenfibrose gelten als Risikofaktoren für das Lungenkarzinom und sind häufig mit dem Zigarettenrauchen assoziiert. Aber auch bei nie-rauchenden Frauen mit benignen Lungenerkrankungen (Asthma, chronische Bronchitis, Emphysem, Pneumonie und Tuberkulose) wurde eine höhere Lungenkrebswahrscheinlichkeit entdeckt.

Eine positive Familienanamnese [19] erhöht bei rauchenden und nicht rauchenden Frauen das Lungenkrebsrisiko erheblich [38]. Bei taiwanesischen nicht rauchenden Frauen erhöhte eine Familienanamnese von Lungenkrebs das Lungenkrebsrisiko um das 5-Fache $[39,40]$ besonders, wenn der Familienangehörige weiblich war. Diese Assoziation war am stärksten bei Frauen unter 60 Jahre und mit Adenokarzinom ausgeprägt. Auch Zwillingsstudien weisen auf eine genetische Lungenkrebs-Disposition speziell bei Frauen hin. Nicht rauchende Frauen in nicht rauchenden Familien mit einer Lungenkrebsanamnese haben ein höheres Risiko als nicht rauchende Männer mit der gleichen Familienanamnese [33].

\section{Treiber-Mutationen bei Nichtrauchern, spezifische Genmutationen, EGFR-Mutation}

Die molekulare Basis der Krebsentstehung kann bei Nichtrauchern und Rauchern unterschiedlich sein. Biologische Faktoren wie Genmutationen im Tumor werden als Prognosefaktoren und als Angriffspunkte für zielgerichtete Therapien immer wichtiger. Der „epidermal growth factor receptor“ EGFR ist bei nichtkleinzelligen Lungenkarzinomen (NSCLC) häufig überexprimiert und eine der „Treiber-Mutationen“ bei der Tumorentwicklung des NSCLC. Diese Entdeckung führte zur Entwicklung zielgerichteter Therapien, die z.B. am EGFR angreifen und die Rezeptor TyrosinKinasen (TKI) hemmen. Es zeigte sich ein Zusammenhang zwischen aktivierenden Mutationen des EGFR-Gens und einer erfolgreichen Therapie mit EGFR-Inhibitoren. Phase-III-Studien [41 -45] zeigten bei Patienten mit EGFR-Mutationen eine hohe Ansprechrate von $60-70 \%$, ein langes progressionsfreies Überleben von 9-13 Monaten und auch ein deutlich längeres Gesamtüberleben als NSCLC-Patienten ohne aktivierende EGFR-Mutation.

\section{EGFR-Mutation}

Schon in ersten retrospektiven Studien fiel auf, dass unter einer EGFR-TKI-Therapie mit Gefitinib das weibliche Geschlecht, Adenokarzinome, Nichtraucherstatus und fernöstliche Herkunft mit einem guten Ansprechen auf die EGFR-TKI-Therapie assoziiert waren $[46,47]$. Auch in 2. Linie hatten, verglichen mit Rauchern oder Ex-Rauchern, die Nie-Raucher unter unselektionierten NSCLC-Patienten unter Erlotinib oder Gefitinib höhere Ansprechraten und bessere Überlebenszeiten $[48,49]$.

Aktivierende EGFR-Mutationen finden sich wesentlich häufiger bei nicht rauchenden Frauen als bei Männern. Sie kommen bei $10-15 \%$ der Lungenkarzinome vor, meist bei nichtplattenepithelialen Tumoren, häufig bei Frauen und Asiaten.

Stratifiziert nach Raucherstatus fand sich [50] aber bei 1467 Nichtrauchern kein Geschlechtsunterschied bei der Häufigkeit der EGFR-Mutation, d.h. man findet sie bei nicht rauchenden Männern genauso häufig wie bei nicht rauchenden Frauen.

Eine Studie der Universität Shanghai an 52 NSCLC [51] an ostasiatischen nie-rauchenden Frauen mit Adenokarzinom ergab bei $90 \%$ Genmutationen an EGFR, KRAS, und anderen (41 EGFR Mutationen, 3 EML4-ALK Fusionen, 2 HER2 Insertionen, 1 KRAS Mutation). Bei 4 Tumoren fanden sich PIK3CA-Mutationen, alle gemeinsam mit EGFR-Mutationen.

EGFR-Genmutationen treten bei Patienten mit Passiv-Zigarettenrauchexposition signifikant seltener auf als bei Nichtrauchern (38,5 vs. $61,4 \%$; P 0,008 ). Passivrauchen ist somit negativ assoziiert mit der Häufigkeit von EGFR-Mutationen bei Nie-Rauchern mit NSCLC [52].

Unter der Drittlinientherapie mit den EGFR-TKI Gefitinib oder Erlotinib in einer fernöstlichen Population aus Taiwan [53] ergab eine Registerstudie gleiche Überlebenszeiten bei Männern und Frauen.

\section{Vergleich Männer und Frauen mit EGFR-Mutation $\nabla$}

Die Studien, die Therapien bei Patienten mit EGFR-Mutationen vergleichen, haben meistens in der Mehrzahl Frauen eingeschlossen. An japanischen Patienten mit EGFR-Mutation und dem Vergleich einer TKI-Therapie oder Chemotherapie [43] ergab die Subgruppenanalyse ein für Frauen gering längeres PFS und ein geringeres Sterberisiko als für Männer (median, 6.5 vs. 6.0 months; HR [hazard ratio for death or disease progression], 0.68). Die Analyse der chinesischen Daten [44] ergab bei 85 Patienten mit EGFR-Mutation keinen Unterschied im progressionsfreien Überleben zwischen Männern und Frauen. Beim Vergleich mit dem neuen irreversiblen EGFT-TKI Afatinib versus Cis-Pem profitierten die Frauen mit EGFR-Mutation mehr vom Afatinib (HR 0,51 vs. 0,61) als die Männer [54]. Zusammenfassend scheinen Frauen mit aktivierender EGFR-Mutation unter einer EGFRTKI-Therapie im Vergleich zu Männern einen leichten Vorteil aufzuweisen.

\section{Anti-VEGF, Anti-EGFR \\ $\nabla$}

Der „Vascular endothelial growth factor“ (VEGF) wirkt pro-angiogen und damit wachstumsfördernd und ist beim NSCLC überexprimiert. VEGF wird von dem Antikörper Bevacizumab angegriffen und verbessert, zusätzlich zur Chemotherapie gegeben, die Ergebnisse beim NSCLC. Unter dieser zielgerichteten Therapie mit Anti-VEGF (Bevacizumab) plus Chemotherapie zeigte die 
E4599-Studie [55] einen 2-monatigen Überlebensvorteil, allerdings nur bei Männern (11,7 Monate vs. 8,7 Mon. $P=0,001$ ). Frauen überlebten unter der Chemotherapie mit oder ohne Bevacizumab gleich lang mit 13,3 oder 13,1 Monaten [56]. Unerwünschte Wirkungen hingegen waren häufiger bei Frauen als bei Männern. Die FLEX-Studie, die eine Chemotherapie mit oder ohne den EGFR-Antikörper Cetuximab verglich, fand, geschlechtsbezogen ausgewertet, einen Überlebensvorteil für Frauen mit Chemotherapie und Cetuximab [57].

\section{Andere Genmutationen}

Das Tumor-Suppressor-Gen P53 ist bei 90\% der kleinzelligen Lungenkarzinome (SCLC) und bei $40-70 \%$ der nichtkleinzelligen Lungenkarzinome (NSCLC) mutiert und zwar häufiger bei Frauen als bei Männern [58]. Lungenkarzinome bei rauchenden Frauen haben häufiger die Tabak-assoziierten P-53-Mutationen als bei Männern: 36\% bei weiblichen und 27\% bei männlichen Rauchern [50]. Die gleiche Mutation fand bei sich bei Nichtrauchern mit Lungenkrebs seltener bei Frauen (13\%) als bei Männern (31\%) und lässt einen größeren Zigarettenrauch-induzierten Schaden beim weiblichen als beim männlichen Geschlecht vermuten.

Neben Amplifikationen [59] werden beim Lungenkarzinom vor allem Punktmutationen beobachtet, zu denen als häufigste die K-RAS-Mutationen gehören. Das K-RAS-Gen gehört zu den ProtoOnkogenen und kommt beim nichtkleinzelligen Karzinomen in $10-15 \%$, am häufigsten beim Adenokarzinom mit $20-30 \%$ vor. K-RAS-Mutationen treten meist bei Rauchern auf und finden sich in Lungentumoren von rauchenden Frauen mit 26\% häufiger als in denen der rauchenden Männer mit 17\% [60]. Der Nachweis dieser Mutation hat therapeutische Relevanz, weil er mit einer primären Resistenz gegenüber einer Therapie mit EGFR-Antagonisten vergesellschaftet ist und auf eine schlechtere Prognose hinweisen kann. Möglicherweise induziert der Zigarettenrauch die K-RAS-Mutation und fördert gemeinsam mit einem wachstumsfördernden Effekt der Östrogene bei Frauen die Entwicklung des Adenokarzinoms.

Die EML4-ALK-Translokation kommt bei circa 3\% aller Adenokarzinome [61] vor und findet sich häufiger bei Frauen als bei Männern. Hier ist eine gezielte Therapie mit dem ALK-Inhibitor Crizotinib möglich.

Eine HER2-Überexpression findet sich bei ca. $2 \%$ der nicht-Plattenepithelkarzinome und scheint mit einer ungünstigen Prognose beim Lungenkarzinom assoziiert [62,63]. Bei resezierten Lungentumoren war eine hohe HER2-Genexpression bei Frauen ein signifikant negativer prognostischer Faktor für das Krankheitsüberleben, aber nicht bei Männern [64]. Therapiestudien mit einer gegen den HER2-Rezeptor gerichteten Therapie $[65,66]$ an unselektionierten Patienten zeigten widersprüchliche Ergebnisse, wohl, weil nur ein geringer Prozentsatz von HER2-positiven Tumoren behandelt wurde [67].

BRAF-Mutationen kommen bei ca. 5\% der Adenokarzinome vor, die V600E- und die Non-V600-Mutation. V600E-Mutationen treten signifikant häufiger bei Frauen 8,6\% als bei Männern 0,9\% auf. Die V600E-Mutationen kommen signifikant häufiger bei Frauen vor und gelten als negative prognostische Faktoren [68].

\section{Unterschiedliche Verteilung von Prognosefaktoren}

Hohe ERCC1, Her2 - und RXR-Spiegel waren für Frauen günstig prognostisch, nicht bei Männern [69]. Bei Männern, aber nicht bei Frauen, war eine niedrige Cyclooxygenase-2-Expression und hohe Ornithin-Carboxylase-Expression mit einem besseren Überleben assoziiert. Weitere Studien sollten klären, ob es sich um prognostische oder prädiktive Faktoren handelt.

\section{Hormonelle Faktoren}

Naheliegend bei der Diskussion um Geschlechterunterschiede beim Lungenkarzinom ist der Blick auf hormonelle Einflüsse. Östrogen fördert die Differenzierung und Reifung der normalen Lunge. Lungenkarzinomzelllinien lassen sich durch Östrogen stimulieren.

Hormonelle Einflüsse auf das Lungenkarzinom werden widersprüchlich diskutiert. Man postuliert einen protektiven Effekt der Östrogene, aber auch eine Lungenkrebszunahme unter Hormonsubstitution [70]. Die „Women's Health Initiative (WHI)“Studie beobachtete Frauen unter der Hormonersatztherapie mit Östrogen und Progestin im Vergleich zu Placebo. Mehr Frauen in der Hormonersatz-Gruppe erkrankten und starben am nichtkleinzelligen Lungenkrebs als in der Placebogruppe [71], was die Hypothese unterstützt, dass weibliche Hormone, wie auch die Hormonersatztherapie (HRT) das Lungenkrebswachstum fördern könnten.

Frauen mit Lungenkarzinom haben ein anderes p53-Mutationsspektrum und eine höhere Frequenz von K-RAS Mutationen als Männer mit Lungenkarzinom. Möglicherweise sind Östrogene und Zigarettenrauch Kofaktoren in der Lungenkarzinomgenese bei Frauen [72]. Zigarettenrauch stimuliert den Metabolismus von 17ß-Estradiol (E 2) in toxische Metaboliten, die mit der DNA in Tumor-Genen interagieren. E(2) stimuliert die Zellproliferation und unterstützt das Tumorwachstum.

In einer klinischen Studie wurden Fulvestrant und Gefitinib [73] bei postmenopausalen Frauen kombiniert gegeben. Fulvestrant sollte das Östrogen-stimulierte Tumorwachstum blockieren. Die Kombination zeigte Effekte, ohne dass Korrelationen zwischen der Östrogenrezeptordichte und dem Ansprechen gefunden werden konnten.

Ein Vergleich unter Berücksichtigung des Menopausenstatus deutete darauf hin, dass die Prognose besonders bei Frauen in der Menopause (älter als 60 Jahre) besser als bei jüngeren Frauen sein könnte [74].

\section{Bessere Lungenkrebs-Prognose bei Frauen Histologie}

Frauen, egal ob rauchend oder nicht rauchend, haben häufiger Adenokarzinome als Männer [10]; bei Frauen ist das bronchioloalveoläre Karzinom 2- bis 4-mal so häufig wie bei Männern [75]. Gegenwärtig findet sich das Adenokarzinom deutlich häufiger bei 41,4\% der Frauen und weniger häufig, 34,1\%, bei Männern.

\section{Metaanalyse-Histologie}

Eine Metaanalyse aus 39 Studien mit 86800 Patienten in allen Stadien des NSLC [76] verglich das Überleben von Männern und Frauen mit NSCLC und fand ein signifikant besseres Überleben der Frauen (HR 0,78), bezogen auf die Patientengruppen mit wenigen Patienten im Stadium I, bei Einschluss von weniger als 50\% Adenokarzinome und unter Berücksichtigung des Raucherstatus. Frauen lebten insgesamt länger als Männer. Nicht der Anteil der Adenokarzinome führte zum besseren Überleben der Frauen. Auch unter Berücksichtigung des Nikotinkonsums zeigte sich, dass der Überlebensvorteil der Frauen unabhängig von deren Rauchgewohnheiten war. Alle Ergebnisse der Metaanalyse bestätigten das bessere Überleben der Frauen mit Lungenkarzinom. 
Nach adjuvanter Chemotherapie im Stadium II und III zeigte sich ein Überlebensvorteil nur bei Frauen mit nicht-plattenepithelialer Histologie [77]. Besonders das bei Frauen häufigere lokalisierte bronchioloalveoläre Karzinom mit seiner geringeren Aggressivität könnte zum besseren Überleben der Frauen nach kurativer Operation beitragen. Die Metaanalyse [76] aber bestätigt den Überlebensvorteil für Frauen, auch wenn in Studien weniger als $50 \%$ Adenokarzinome eingeschlossen waren, sodass der Einfluss der Histologie nicht wesentlich zu sein scheint.

\section{Klinische Erfahrungen}

Viele Studien ergaben für Frauen mit Lungenkarzinom ein besseres Überleben als für Männer: Schon länger ist das weibliche Geschlecht als unabhängiger Prognosefaktor für das Überleben beschrieben worden.

\section{a) Registerstudien}

Schon in den 70er-Jahren zeigte sich, dass Frauen mit fortgeschrittenem zytostatisch behandeltem Adenokarzinom oder SCLC länger als Männer lebten [78]. Monozentrisch wurden 7500 Patienten im Zeitraum 1997-2000 bezüglich des Überlebens beim NSCLC ausgewertet [79]: Die Frauen lebten mit durchschnittlich 12,4 Monaten signifikant länger als die Männer mit 10,3 Monaten, unabhängig vom Erkrankungsstadium.

Eine Analyse an 19000 Patienten über 65 Jahren in den USA ergab für ältere Frauen in allen Stadien und unter allen TherapieGruppen ein um ca. 25\% signifikant besseres Lungenkrebs-spezifisches Überleben als für Männer, unabhängig von der angewandten Therapie. Der Vorteil für Frauen wurde nur bei Adenooder großzelligem NSCLC, nicht bei Plattenepithelkarzinomen, beobachtet. Auch unbehandelte Frauen hatten ein besseres Überleben, was auf eine unterschiedliche Tumorbiologie bei Frauen hinweisen könnte [80].

In 3 retrospektiven japanischen Studien mit Resektionen in den Jahren 1989, 1994 und 1999 war das Überleben der Frauen in allen Zeitperioden und Stadien besser [81]. Bei den Frauen wurde ein höherer Anteil gut differenzierter, langsam wachsender Adenokarzinome beobachtet, die zur bei Frauen besseren Prognose beigetragen haben könnten.

\section{b) NSCLC: Frühe Stadien}

Beim operierten NSCLC an 1242 Patienten erfolgten [82] zwar bei Frauen weniger häufig komplette Resektionen (79,6 vs. 85,2\%), aber nach R0-Resektion war das Überleben bei Frauen im Alter über 60 Jahre besser als bei Männern.

Viele Beobachtungen deuten auf einen Überlebensvorteil für Frauen nach Resektion in frühen Stadien mit nicht-Plattenepithel-CA hin, möglicherweise auch, weil hier der Anteil an frühen bronchioloalveolären Karzinomen höher als bei den Männern war.

Auch in prospektiven Studien war das weibliche Geschlecht ein Prognosefaktor für das Überleben [83] mit einer niedrigeren Sterbewahrscheinlichkeit bei Frauen und einer niedrigeren Morbidität und Mortalität bei der Resektion des Lungenkarzinoms.

Eine prospektive Studie an 1085 Pat. zeigte Überlebensvorteile für Frauen im Stadium I- III mit NSCLC [84]. Frauen waren signifikant jünger, hatten häufiger Adenokarzinome und wurden in früheren Stadien diagnostiziert als Männer. Das alters- und stadienadjustierte 5-Jahres-Überleben (60 vs. 50\%) war bei Frauen signifikant besser: Im Stadium I überlebten 69\% Frauen vs. $64 \%$
Männer 5-Jahre, im Stadium II 60\% Frauen gegenüber 50\% Männer und auch im Stadium III war das 5-Jahres-Überleben der Frauen mit 46 vs. 37\% besser. Nach neoadjuvanter Chemotherapie hatten Frauen signifikant höhere Ansprechraten als Männer. Gemäß einer US Register-Analyse an 129000 Patienten, hiervon nur 5\% Frauen, hatten die Frauen mit NSCLC gemäß Multivarianzanalyse [85] nach OP eine geringere Mortalität und Morbidität. Auch unter Berücksichtigung von Rasse und Einkommen blieb das weibliche Geschlecht ein unabhängiger günstiger Prognosefaktor mit einer um $24 \%$ geringeren Mortalität. Weibliches Geschlecht (46,2 vs. 32,0\%, p<0,001), afroamerikanische Herkunft $(8,3$ vs. $4,1 \%, p=0,03)$ und ein höheres Einkommen $(34,9$ vs. $25,9 \%, p=0,01)$ waren häufiger bei Überlebenden.

Nach neoadjuvanter Chemotherapie zeigte sich im Stadium III N2-NSCLC ein Überlebensvorteil für Frauen, und auch unter der Bestrahlung ergab sich in den RTOG-Studien mit Strahlen und kombinierter Chemo-Strahlentherapie für Frauen mit 11,4 Monaten ein besseres Überleben als für Männer mit 9,9 Monaten [86].

\section{NSCLC Lokal fortgeschritten oder metastasiert}

In Therapiestudien beim fortgeschrittenen NSCLC ist das weibliche Geschlecht ein günstiger Prognosefaktor. Bei der Analyse von 2500 Patienten in 13 SWOG-Studien beim fortgeschrittenen NSCLC der Jahre 1974-1987 war das weibliche Geschlecht ein unabhängiger prognostisch günstiger Faktor [87]. Medianes Überleben und 1-Jahres-Überleben waren bei Frauen signifikant besser als bei Männern: 1-J-ÜL: 19\% bei Frauen und 14\% bei Männern.

Eine Metaanalyse von 1365 Patienten aus 9 prospektiven RTOG (Radiation Therapy Oncology Group) Studien [88] zeigte für Männer eine signifikant höhere Mortalität als für Frauen. Rasse und Lebensform waren keine unabhängigen Variablen für das Überleben. Allein lebende Frauen hatten ein signifikant besseres Überleben als allein lebende Männer (HR 0,72), und verheiratete Männer hatten ein schlechteres Überleben als allein lebende Frauen (HR 1,36). Die Studie zeigte, dass, obwohl es einige Einflussgrößen wie Geschlecht, Rasse und Lebensform gibt, das weibliche Geschlecht der Prognosefaktor mit der höchsten Signifikanz bei nicht-operablen NSCLC-Patienten ist.

Unter Strahlentherapie [89] in den frühen Stadien I-III fand sich an 831 NSCLC-Patienten (319 Frauen, 512 Männer) ein Vorteil zugunsten von Frauen: Frauen hatten frühere Stadien, weniger geraucht (häufiger $<50$ pack-years) und häufiger Adeno- oder großzellige Karzinome. Obwohl sich die Therapie nicht zwischen Männern und Frauen unterschied, war das 5-Jahres-Überleben der Frauen signifikant besser als das der Männer. Das rezidivfreie 5-Jahres-Überleben betrug bei Frauen 31,2 \% und 20,1 \% bei Männern; Fernmetastasen traten bei Frauen bei 37,6\% auf, bei Männern in 48,8\%. Ungünstige Prognosefaktoren waren männliches Geschlecht, Gewichtsverlust, Alter über 65 Jahre und Stadium III. Ähnliche Ergebnisse fanden sich in Europa für das NSCLC unter cisplatinhaltiger Chemotherapie, indem das weibliche Geschlecht als unabhängiger günstiger Prognosefaktor [90] identifiziert wurde. Die EGOG-Studie E1594 [91], die vier verschiedene Chemotherapie-Regime beim fortgeschrittenen NSCLC verglich und keinen Überlebensunterschied zwischen den Therapiearmen fand, zeigte nach geschlechtsbezogener Analyse [92,93] bei gleichen Ansprechraten einen 2-monatigen Überlebensvorteil für Frauen (med. Überleben 9,2 Monate gegenüber 7,3 Monate bei Männern) (๑ Tab.3). Andere Risikofaktoren, wie Allgemeinzustand, Gewichtsverlust oder Häufigkeit von Hirnmetastasen unterschieden 
Tab.3 Überlebensunterschied nach Geschlecht E1594-Studie [91].

\begin{tabular}{|llll} 
& Frauen & Männer & p-Wert \\
\hline Ansprechrate & $19 \%$ & $19 \%$ & 0,15 \\
\hline med. Überleben/Mon. & 9,2 & 7,3 & 0,0004 \\
\hline
\end{tabular}

sich nicht zwischen Männern und Frauen. Die Therapie-Toxizität war bei Frauen höher als bei Männern. Ein weiterer Vergleich von Patienten aus SWOG-Studien bestätigte den Überlebensvorteil der Frauen mit einem um 3 Monate längeren Überleben als bei Männern (11 vs. 8 Monate), ohne dass Toxizitätsunterschiede zwischen den Geschlechtern beobachtet wurden [94].

In vielen randomisierten Chemotherapie-Studien im Stadium IV und auch in Studien zur Zweitlinientherapie ist das weibliche Geschlecht ein günstiger Prognosefaktor mit jeweils besserem Ansprechen und besserem Überleben.

\section{Kleinzelliges Lungenkarzinom \\ $\nabla$}

Auch beim kleinzelligen Lungenkarzinom (SCLC) ist das weibliche Geschlecht ein günstiger Prognosefaktor [95]. Man fand beim fortgeschrittenen SCLC bei Frauen mehr Langzeitüberlebende als bei Männern [96]. Eine Analyse von 10 SWOG-Studien beim LD und ED-SCLC ergab beim LD-SCLC bei Frauen ein signifikant längeres mittleres Überleben von 24,4 Monaten gegenüber 17,7 Monaten bei Männern. Beim fortgeschrittenen SCLC hingegen zeigte sich kein Vorteil für Frauen [97]. Eine Multivariatanalyse zur Toxizität zeigte für Frauen eine erhöhte Toxizität mit mehr Therapieverschiebungen, aber einem signifikant besseren Ansprechen von 80,3\% bei Frauen gegenüber 66,9\% bei Männern. Auch hatten die Frauen ein signifikant besseres medianes Überleben mit 1,3 Jahren gegenüber 0,91 Jahren bei Männern [98]. Diese Beobachtungen führten dazu, dass in später folgenden SCLC-Studien auch nach Geschlecht stratifiziert wurde.

Zusammenfassend fand sich in vielen Therapiestudien beim kleinzelligen und nichtkleinzelligen Lungenkarzinom regelmäßig ein Überlebensvorteil für die Frauen. Insbesondere findet sich in der Kohorte der nicht rauchenden Patientengruppe ein größerer Anteil an Frauen mit definierten genetischen Varianten, die als zielgerichtete Therapieoption ggf. genutzt werden können. Das weibliche Geschlecht gilt in Therapiestudien als etablierter günstiger Prognosefaktor.

\section{Interessenkonflikt}

Die Autoren geben an, dass kein Interessenkonflikt besteht.

\section{Literatur}

1 Malvezzi M, Arfe A, Bertuccio P. European cancer mortality predictions for the year 2011. Ann Oncol 2011; 22: 947-956

2 Jemal A, Siegel $R$, Ward $E$ et al. Cancer statistics 2004. CA Cancer J Clin 2006; 56: $106-130$

3 Patel JD. Lung Cancer in Women. J Clin Oncol 2005; 23: 3212 - 3218

4 American Cancer Society. Cancer facts and figures. Atlanta, GA: American Cancer Society; 2010

5 Thun MJ, Carter BD, Feskanich D. 50-Year Trends in Smoking-Related Mortality in the United States. N Engl J Med 2013; 368: 4

6 Haiman CA, Stram DO, Wilkens LR et al. Ethnic and racial differences in the smoking-related risk of lung cancer. N Engl J Med 2006; 354: 333 342
7 Robert Koch-Institut und die Gesellschaft der epidemiologischen Krebsregister in Deutschland e.V. Hrsg. Krebs in Deutschland 2007/ 2008. 8. Ausgabe. Berlin: 2012

8 Jemal A, Thun MJ, Ries LA et al. Annual report to the nation on the status of cancer, 1975-2005, featuring trends in lung cancer, tobacco use, and tobacco control. J Natl Cancer Inst 2008; 100: 1672 - 1694

9 World Health Organization. Women and health: today's evidence, tomorrow's agenda. Geneva, Switzerland: World Health Organization Press; 2009

10 Klingerman $S$, White $C$. Epidemiology of Lung Cancer in Women: Risk Factors, Survival, and Screening. AJR 2011; 196: 287 - 295

11 Pirie K, Peto R, Reeves GK et al. The 21 st century hazards of smoking and benefits of stopping: a prospective study of one million women in the UK. Lancet 2013; 381: 133-141

12 Laue E. Statistisches Bundesamt Wiesbaden; 2010

13 Risch HA, Howe GR, Jain M et al. Are female smokers at higher risk for lung cancer than male smokers? A case-control analysis by histologic type Am J Epidemiol 1993; 138: 281 - 293

14 Feskanich D, Ziegler RG, Michaud DS et al. Prospective study of fruit and vegetable consumption and risk of lung cancer among men and women. J Natl Cancer Inst 2000; 92: 1812-1823

15 Henschke CI, Yip R, Miettinen OS. International Early Lung Cancer Action Program Investigators. Women's susceptibility to tobacco carcinogens and survival after diagnosis of lung cancer. JAMA 2006; 296 : $180-184$

16 Ryu JS, Jeon SH, Kim JS. Gender Differences in Susceptibility to Smoking among Patients with Lung Cancer. Korean J Intern Med 2011; 26: 427 431

17 Freedman ND, Leitzmann MF, Hollenbeck AR et al. Cigarette smoking and subsequent risk of lung cancer in men and women: analysis of a prospective cohort study. Lancet Oncol 2008; 9: 649-656

18 Bain C, Feskanich D, Speizer F et al. Lung cancer rates in men and women with comparable histories of smoking. J Natl Cancer Inst 2004; 96: $826-834$

19 Subramanian J, Govindan R. Lung cancer in never smokers: A review. J Clin Oncol 2007; 25: 561 - 570

20 Gorlova OY, Weng SF, Zhang Yet al. DNA repair capacity and lung cancer risk in never smokers. Cancer Epidemiol Biomarkers Prev 2008; 17: $1322-1328$

21 Wei $Q$, Cheng L, Amos CI et al. Repair of tobacco carcinogen-induced DNA adducts and lung cancer risk: a molecular epidemiologic study. J Natl Cancer Inst 2000; 92: $1764-1772$

22 Planchard D, Loriot Y, Goubar A et al. Differential expression of biomarkers in men and women. Semin Oncol 2009; 36: $553-565$

23 Mollerup S, Ryberg D, Hewer A et al. Sex differences in lung CYP1A1 expression and DNA adduct levels among lung cancer patients. Cancer Res 1999; 59: 3317 - 3320

24 Bell D, Taylor J, Paulson D et al. Genetic risk and carcinogen exposure:a common inherited defect of the carcinogen-metabolism gene glutathione S-transferase M1 (GSTM1) that increases susceptibility to bladder cancer. J Natl Cancer Inst 1993; 85: 1159-1164

25 Dresler C, Fratelli C, Babb J et al. Gender differences in genetic susceptibility for lung cancer. Lung Cancer 2000; 30: 153-160

26 Hackshaw AK, Law MR, Wald NJ. The accumulated evidence on lung cancer and environmental tobacco smoke. BMJ 1997; 315: 980 - 988

27 Öberg M,Jaakkola MS, Woodward A. Worldwide burden of disease from exposure to second-hand smoke: a retrospective analysis of data from 192 countries. The Lancet 2011; 377, 760: 139-146

28 Tredaniel J, Boffetta P, Saracci $R$ et al. Non-smoker lung cancer deaths attributable to exposure to spouse's environmental tobacco smoke. Int J Epidemiol 1997; 26: 939 - 944

29 Vineis P, Alavanja M, Buffler P et al. Tobacco and cancer: Recent epidemiological evidence. J Natl Cancer Inst 2004; 96: 99-106

30 World Health Organization. WHO Report on the Global Tobacco Epidemic. Geneva, Switzerland: World Health Organization Press; 2008

31 Sun S, Schiller JH, Gazdar AF. Lung cancer in never smokers - a different disease. Nat Rev Cancer 2007; 7: 10778-790

32 Toh CK. Never smokers with lung cancer. J Clin Oncol 2006; 24: 2245 2251

33 Wakelee HA, Chang ET, Gomez SL et al. Lung cancer incidence in never smokers. J Clin Oncol 2007; 25: $472-478$

34 Kawaguchi T, Takada M, Kubo A. Gender, Histology, and Time of Diagnosis Are Important Factors for Prognosis. Analysis of 1499 NeverSmokers with Advanced Non-small Cell Lung Cancer in Japan. J Thorac Oncol 2010; 5: $1011-1017$ 
35 Nordquist LT, Simon GR, Cantor A et al. Improved survival in never-smokers vs current smokers with primary adenocarcinoma of the lung. Chest 2004; 126: 347-351

36 Field RW, Steck DJ, Smith BJ et al. Residential radon gas exposure and lung cancer: the Iowa Radon Lung Cancer Study. Am J Epidemiol 2000; 151: $1091-1102$

$37 \mathrm{Ko} Y C$, Cheng $L S$, Lee $C H$ et al. Chinese food cooking and lung cancer in women nonsmokers. Am J Epidemiol 2000; 151: 140-147

38 Matakidou A, Eisen T, Houlston RS. Systematic review of the relationship between family history and lung cancer risk. Br J Cancer 2005; 93: $825-833$

$39 \mathrm{Wu}$ A, Fontham E, Reynolds $P$ et al. Family history of cancer and risk of lung cancer among lifetime nonsmoking women in the United States. Am J Epidemiol 1996; 143: 535-542

$40 \mathrm{Wu}$ PF, Lee CH, Wang MJ et al. Cancer aggregation and complex segregation analysis of families with female non-smoking lung cancer probands in Taiwan. Eur J Cancer 2004; 40: 260 - 266

41 Mok TS, Wu YL, Thongprasert S et al. Gefitinib or carboplatin-paclitaxel in pulmonary adenocarcinoma. N Engl J Med 2009; 361: 947-957

42 Mitsudomi T, Morita S, Yatabe Yet al. Gefitinib versus cisplatin plus docetaxel in patients with non-small-cell lung cancer harbouring mutations of the epidermal growth factor receptor (WJ-TOG3405): an open label, randomised phase 3 trial. Lancet Oncol 2010; 11: 121 - 128

43 Maemondo M, Inoue A, Kobayashi Ket al. Gefitinib or chemotherapy for nonsmall-cell cancer with mutated EGFR. N Engl J Med 2010; 362: $2380-2388$

44 Rosell R, Carcereny E, Gervais $R$ et al. Erlotinib versus standard chemotherapy as first-line treatment for European patients with advanced EGFR mutation-positive non-small-cell lung cancer (EURTAC): a multicentre, open-label, randomised phase 3 trial. Lancet Oncol 2012; 13: $239-246$

45 Zhou C, Wu YL, Chen $G$ et al. Erlotinib versus chemotherapy as first-line treatment for patients with advanced EGFR mutation-positive nonsmall-cell lung cancer (OPTIMAL, CTONG-0802): a multicenter, openlabel, randomized, phase 3 study. Lancet Oncol 2011; 12: 735-742

46 Kris MG, Natale RB, Herbst RS. Efficacy of gefitinib, an inhibitor of the epidermal growth factor receptor tyrosine kinase, in symptomatic patients with non-small-cell lung cancer: a randomized trial. JAMA 2003; 290: 2149-2158

47 Herbst RS, Giaccone G, Schiller J. Subset analysis of INTACT results for gefitinib (ZD1839) when combined with platinum based chemotherapy for advanced non-small-cell lung cancer (Abstract). Proc Am Soc Clin Oncol 2003; 22: 627

48 Thatcher N, Chang A, Parikh P et al. Gefitinib plus best supportive care in previously treated patients with refractory advanced non-small-cell lung cancer: Results from a randomised, placebocontrolled, multicentre study (Iressa Survival Evaluation in Lung Cancer). Lancet 2005; 366: $1527-1537$

49 Shepherd FA, Rodrigues Pereira J, Ciuleanu T et al. Erlotinib in previously treated non-small-cell lung cancer. N Engl J Med 2005; 353: 123-132

50 Toyooka S, Tsuda T, Gazdar AF. The TP53 gene, tobacco exposure, and lung cancer. Hum Mutat 2003; 21: 229-239

51 Sun $Y$, Ren $Y$, Fang $Z$ et al. Lung Adenocarcinoma From East Asian Never-Smokers Is a Disease Largely Defined by Targetable Oncogenic Mutaal.nt Kinases. J Clin Oncol 2010; 28: 4616-4620

52 Lee YJ, Cho BC, Jee SH et al. Impact of Environmental Tobacco Smoke on the Incidence of Mutations in Epidermal Growth Factor Receptor Gene in Never-Smoker Patients With Non-Small-Cell Lung Cancer. J Clin Oncol 2009; 28: 487-492

53 Shao YY, Shau WY, Lin ZZ. Comparison of gefitinib and erlotinib efficacies as third-line therapy for advanced non-small-cell lung cancer. Eur J Cancer 2013; 49 (Suppl. 01): 106-114

54 Yang JCH, Schuler MH, Yamamoto N. LUX-Lung 3: A randomized, openlabel, phase III study of afatinib versus pemetrexed and cisplatin as first-line treatment for patients with advanced adenocarcinoma of the lung harboring EGFR-activating mutations. J Clin Oncol 2012; 30: abstr LBA7500

55 Sandler A, Gray R, Perry MC et al. Paclitaxel-carboplatin alone or with bevacizumab for non-small-cell lung cancer. N Engl J Med 2006; 355 : $2542-2550$

56 Brahmer JR, Dahlberg SE, Gray RJ et al. Sex differences in outcome with bevacizumab therapy: analysis of patients with advanced-stage nonsmall cell lung cancer treated with or without bevacizumab in combination with paclitaxel and carboplatin in the Eastern Cooperative Oncology Group Trial 4599. J Thorac Oncol 2011; 6: 103-108
57 Pirker R, Pereira JR, Szczesna A et al. Cetuximab plus chemotherapy in patients with advanced non-small-cell lung cancer (FLEX): an open-label randomised phase III trial. Lancet 2009; 373: 1525 - 1531

58 Kure EH, Ryberg D, Hewer $A$ et al. p53 mutations in lung tumours: relationship to gender and lung DNA adduct levels. Carcinogenesis 1996; 17: $102201-2205$

59 Petersen I. Morphologische und molekulare Diagnostik des Lungenkarzinoms. Dtsch Arztebl Int 2011; 108: 525-531. DOI 10.3238/arztebl.2011.0525

60 Nelson HH, Christiani DC, Mark EJ et al. Implications and prognostic value of K-ras mutation for early-stage lung cancer in women. J Natl Cancer Inst 1999; 91: 2032-38

61 Shaw AT, Yeap BY, Mino-Kenudson M. Clinical Features and Outcome of Patients With Non-Small-Cell Lung Cancer Who Harbor EML4-ALK. JCO 2009; 27, 26: 4247-4253

62 Meert AP, Martin B, Paesmans M et al. The role of HER-2/neu expression on the survival of patients with lung cancer: a systematic review of the literature. Br J Cancer 2003; 89: 959-965

63 Nakamura H, Kawasaki N, Taguchi M et al. Association of HER-2 overexpression with prognosis in nonsmall cell lung carcinoma: a metaanalysis. Cancer 2003; 103: 1865 - 1873

64 Al-Saad S, Al-Shibli K, Donnem T. Clinical Significance of Epidermal Growth Factor Receptors in Non-small Cell Lung Cancer and a Prognostic Role for HER2 Gene Copy Number in Female Patients. J Thorac Oncol 2010; 5: 1536-1543

65 Rosell $R$. Toward customized trastuzumab in HER-2/neu-overexpressing non-small-cell lung cancers. J Clin Oncol 2004; 22: 1171 - 1173

66 Cappuzzo F, Bemis L, Varella-Garcia M. HER2 mutation and response to trastuzumab therapy in non-small-cell lung cancer. N Engl J Med 2006; 354: $2619-2621$

67 Gatzemeier U, Groth G, Butts C et al. Randomized phase II trial of gemcitabine-cisplatin with or without trastuzumab in HER2-positive nonsmall-cell lung cancer. Ann Oncol 2004; 15: 19-27

68 Marchetti A, Felicioni L, Malatesta S. Clinical Features and Outcome of Patients With Non-Small-Cell Lung Cancer Harboring BRAF Mutations. J Clin Oncol 2011; 29: 3574-3579

69 Dannenberg K, Brabender J, Schneider S et al. Gender differences in the predictive power of prognostic factors in NSCLC (Abstract). Proc Am Soc Clin Oncol 2004; 22: (Abstract 619s)

70 Schabath $M B, W u X$, Vassilopoulou-Sellin $R$ et al. Hormone replacement therapy and lung cancer risk: a case control analysis. Clin Cancer Res 2004; 10: $113-123$

71 Chlebowski RT, Schwartz AG, Wakelee H et al. Women's Health Initiative Investigators. Oestrogen plus progestin and lung cancer in postmenopausal women (Women's Health Initiative trial): a post-hoc analysis of a randomised controlled trial. Lancet 2009; 374: $1243-1251$

72 Gasperino J. Gender is a risk factor for lung cancer. Med Hypotheses 2011; 76: 328-31

73 Traynor AM, Schiller JH, Stabile LP et al. Combination therapy with gefitinib and fulvestrant (G/F) for women with non-small-cell lung cancer (NSCLC) (Abstract). Proc Am Soc Clin Oncol 2005; 23: 165

74 Wakelee HA, Dahlberg SE, Schiller JH et al. Menopausal status of women may affect survival in advanced NSCLC: Analysis of recent Eastern Cooperative Oncology Group (ECOG) studies using age of 60 years or older as a surrogate marker. 12th World Conference on Lung Cancer Proceedings 2007; 2 (Suppl. 04): 570 (Abstr \#P1052)

75 Patel JD, Bach PB, Kris MG. Lung cancer in US women: a contemporary epidemic. JAMA 2004; 291: $1763-1768$

76 Nakamura H, Shinmyo AK, Mochizuki A. Female gender is an independent prognostic factor in non-small-cell lung cancer: a meta-analysis. Annals Thor Cardiovasc Surg 2011; 17: 469-480

77 Keller SM, Vangel MG, Adak S. The influence of gender on survival and tumor recurrence following adjuvant therapy of completely resected stages II and IIIa non-small cell lung cancer. Lung Cancer 2002; 37: 303-309

78 Edmonson JH, Lagakos SW, Selawry OS. Cyclosphosphamide and CCNU in the treatment of inoperable small cell carcinoma and adenocarcinoma of the lung. Cancer Treat Rep 1976; 60: 925 -932

79 Moore $R$, Doherty $D$, Chamberlain $R$ et al. Sex differences in survival in non-small-cell lung cancer patients 1974-1998. Acta Oncol 2004; 43: $57-64$

80 Wisnivesky JP, Halm EA. Sex differences in lung cancer survival: do tumors behave differently in elderly women? J Clin Oncol 2007; 25: $1705-1712$ 
81 Asamura H, Goya T, Koshiishi Y. A Japanese Lung Cancer Registry study: prognosis of 13,010 resected lung cancers. J Thorac Oncol 2008; 3: 4652

82 Minami H, Yoshimura M, Miyamoto Yet al. Lung cancer in women: sexassociated differences in survival of patients undergoing resection for lung cancer. Chest 2000; 118: 1603-1609

83 Visbal AL, Williams BA, Nichols FCIII et al. Gender differences in nonsmall-cell lung cancer survival: an analysis of 4,618 patients diagnosed between 1997 and 2002. Ann Thorac Surg 2004; 78: 209-215

84 Cerfolio RJ, Bryant AS, Scott $E$ et al. Women with pathologic stage I, II, and III non-small cell lung cancer have better survival than men. Chest 2006; 130: $061796-1802$

85 LaPar DJ, Bhamidipati CM, Harris DA. Gender, Race and Socioeconomic Status Affects Outcomes Following Lung Cancer Resections in the United States. Ann Thorac Surg 2011; 92: 434-439

86 Werner-Wasik M, Scott C, Cox JD et al. Recursive partitioning analysis of 1999 Radiation Therapy Oncology Group (RTOG) patients with locallyadvanced non-small-cell lung cancer (LA-NSCLC): identification of five groups with different survival. Int J Radiat Oncol Biol Phys 2000; 48: $1475-1482$

87 Albain KS, Unger J, Gotay CC et al. Toxicity and survival by sex in patients with advanced non-small cell lung carcinoma (NSCLC) on modern Southwest Oncology Group (SWOG) trials. 2007 ASCO Annual Meeting Proceedings (Post-Meeting Edition). J Clin Oncol 2007; 25: 7549

88 Siddiqui F, Bae K, Langer CJ. The Influence of Gender, Race, and Marital Status on Survival in Lung Cancer Patients. Analysis of Radiation Therapy Oncology Group Trials. J Thorac Oncol 2010; 5: 631 -639

89 McGovern SL, Liao Z, Bucci K et al. Is Sex Associated With the Outcome of Patients Treated With Radiation for Nonsmall Cell Lung Cancer? Cancer 2009; 115 (Suppl. 14): 3233-3242

90 Paesmans M, Sculier JP, Libert G. Prognostic factors for survival in advanced non-small-cell lung cancer: univariate and multivariate analyses including recursive partitioning and amalgamation algorithms in 1,052 patients. J Clin Oncol 1995; 13: $1221-1230$

91 Schiller JH, Harrington D, Belani CP et al. Comparison of four chemotherapy regimens for advanced non-small-cell lung cancer. N Engl J Med 2002; 346: 92 - 98

92 Wakelee HA, Wang W, Schiller JH et al. Survival differences by sex for patients with advanced non-small cell lung cancer on Eastern Cooperative Oncology Group trial 1594. J Thorac Oncol 2006; 1: 441 - 446

93 Wakelee HA, Dahlberg SE, Schiller JH et al. Menopausal status of women may affect survival in advanced NSCLC: analysis of recent Eastern Cooperative Oncology Group (ECOG) studies using age of 60 years or older as a surrogate marker. J Thorac Oncol 2007; 2: 570 Abstract P1052

94 Albain KS, Unger J, Gotay CC et al. Toxicity and survival by sex in patients with advanced non-small cell lung carcinoma (NSCLC) on modern Southwest Oncology Group (SWOG) trials. Proc Am Soc of Clin Oncol 2007; 25: 396s Abstract 7549

95 Paesmans M, Sculier JP, Lecomte J et al. Prognostic factors for patients with small cell lung carcinoma: analysis of a series of 763 patients included in 4 consecutive prospective trials with a minimum follow-up of 5 years. Cancer 2000; 89: 523-33

96 Johnson BE, Steinberg SM, Phelps R et al. Female patients with small cell lung cancer live longer than male patients. Am J Med 1988; 85: 194 196

97 Albain KS, Crowley JJ, LeBlanc M et al. Determinants of improved outcome in small-cell lung cancer: an analysis of the 2,580-patient Southwest Oncology Group database. Am J Clin Oncol 1990; 8: 1563 - 1574

98 Singh W, Parulekar N, Murray R et al. Influence of gender on treatment outcome and toxicity in small cell lung cancer (SCLC) (Abstract). Proc Am Soc Clin Oncol 2004; 22: 14 S 